\title{
ORAL METHOTREXATE AS THE SECOND-LINE CHEMOTHERAPY IN PLATINUM- REFRACTORY OR RELAPSED SQUAMOUS CELL CARCINOMA OF THE HEAD AND NECK
}

\author{
Muhammad Irfan ${ }^{1}$, Tahir Mehmood ${ }^{1}$, Arif Jamshed ${ }^{1}$, Irfan Haider ${ }^{1}$, Hassan Iqbal' \\ Raza Hussain ${ }^{2}$ \\ ${ }^{1}$ Department of Radiation Oncology, Shaukat Khanum Memorial Cancer Hospital and Research Centre, Lahore, \\ Pakistan, ${ }^{2}$ Department of Surgical Oncology, Shaukat Khanum Memorial Cancer Hospital and Research Centre, \\ Lahore, Pakistan
}

Received: 2 June 2015 / Accepted: 15 August 2016

\begin{abstract}
Purpose: Platinum-refractory or relapsed squamous cell carcinoma of the head and neck (SCCHN) is considered to have poor prognosis. Although cetuximab is currently recommended as category 1 in this group of patients, the use of it is hampered in low- and middle-income countries (LMICs) like Pakistan due to financial constraints. Further, majority of the population of these countries is unable to tolerate toxicity related to other intravenous chemotherapeutic agents due to lower socioeconomic background with poor nutrition status. The aim of this study is to evaluate the response rate and toxicity of oral methotrexate (MTX) in platinum-refractory or relapsed SCCHN.

Methods: Between June 2008 and December 2012, 71 patients received palliative oral MTX either due to recurrent or persistent disease. With a median age of 51 years (range $22-75$ ), there were $68 \%$ of males and $32 \%$ of females. Site distribution was as following; oral cavity $58 \%$, nasopharynx $25 \%$, hypopharynx $7 \%$, paranasal sinuses $6 \%$, larynx $3 \%$, Oropharynx 1\%. Patterns of recurrence; local 32\%, regional $07 \%$, loco-regional $10 \%$, distant $15 \%$ and persistent disease in $36 \%$ of the patients. All the patients received oral MTX $10 \mathrm{mg}$ once a day, 4 days a week. To contain the possible side effects associated with MTX, folinic acid $15 \mathrm{mg}$ per oral every 6 hourly on day 5 was prescribed. Response assessment was done on two monthly basis. Response, toxicity, mean response time and mean time to progression were determined.

Results: Response to MTX was as following; complete response 3\%, partial response $4 \%$, stable disease $11 \%$ and progressive disease in $82 \%$ of the patients respectively. Toxicity related to oral MTX includes neutropenia Grade III in $1 \%$ while mucositis Grade III in $10 \%$ of the patients, respectively. None of the patient had Grade IV mucositis or haematological toxicity. Treatment was stopped in $13 \%$ of the patients due to poor compliance. Mean response time was 4 months (range $1-20$ ) and mean time to progression was 5 months (range 1-23).

Conclusion: Oral MTX is a simple, cost-effective and well-tolerated regimen to be used on outpatient basis for palliation in platinum-refractory or relapsed SCCHN in LMICs and debilitated patients. This treatment merits further evaluation in large-scale clinical trials.
\end{abstract}

Key words: Oral methotrexate, platinum refractory, relapsed squamous cell carcinoma of head and neck

\section{Introduction}

Squamous cell carcinoma of the head and neck (SCCHN) is the sixth most common cancer in the world, with approximately half million new cases per year. ${ }^{[1]}$ Two-

Correspondence: Dr. Muhammad Irfan, Department of Radiation Oncology, Shaukat Khanum Memorial Cancer Hospital and Research Centre, Lahore, Pakistan.

Email: drmuhammadirfan@hotmail.com thirds of patients present with locally advanced disease and $10 \%$ are metastatic ${ }^{[2]}$ Despite the progress in the primary treatment by combining chemotherapy, surgery, radiation therapy and supportive care, $30-50 \%$ of the patients eventually develop locoregional or distant relapse..$^{[3]}$

Patients with recurrent or metastatic SCCHN have poor prognosis as they have limited treatment options. ${ }^{[4,5]} \mathrm{A}$ few patients with locoregional recurrence can be salvaged 
by surgery or reirradiation. Palliative chemotherapy is considered as standard of care in these patients. Platinumbased regimen with 5-FU is often considered as standard in patients with good performance status. ${ }^{[4,6,7]}$ The other drugs that have been used are methotrexate (MTX), bleomycin, capecitabine and ifosfamide. ${ }^{[8]}$ These drugs produce response rate up to $30 \%$ while introduction of new chemotherapy agents such as taxane can increase response rate up to $43 \% .{ }^{[9]}$ However, none of these drugs as single agent or in combination have been found to prolong survival. ${ }^{[10]}$

The use of cetuximab in combination with cisplatin and 5 FU leads to an improvement in overall survival (OS) with respect to cisplatin and 5FU combination. That is why ESMO and NCCN recommend it as category 1 in recurrent and/or metastatic (R/M) SCCHN..$^{[3,7,11-13]}$

Options are further limited for the patients who fail after platinum-based chemotherapy, and response rates are generally poor (approximately 3\%). ${ }^{[9]}$ Leon et al. ${ }^{[14]}$ investigated the best supportive care for patients with platinum-refractory SCCHN and showed a median survival time of 56 days. Herbst et al. ${ }^{[15]}$ reported that median OS was 4.3 months and response rate was 13\% in platinum-refractory patients treated with cetuximab and cisplatin regimen. Cetuximab is currently considered to be standard of care for this population of patients. However, the utilization of this agent in our country is hampered by the financial and logistic considerations. Other options are taxanes, MTX, bleomycin, capecitabine and ifosfamide. ${ }^{[8,9]}$

In low- and middle-income countries (LMICs) like Pakistan, where head and neck cancer is prevalent in people with low socioeconomic status, the use of cetuximab is not feasible due to financial constraints. Further, majority of the population of these countries with SCCHN is unable to tolerate toxicity related to other intravenous chemotherapeutic agents due to lower socioeconomic background with poor nutrition status. MTX, most economical and least toxic, is a very good alternative for this selected group of patients.

In this retrospective study, we investigated the efficacy and tolerability of oral MTX in platinum-refractory or relapsed SCCHN patients. Oral MTX was given due to the reason that patients were not willing for intravenous MTX or not able to come to hospital on weekly basis from remote areas of the country.

\section{Methods}

Clinical data were obtained retrospectively from hospital information system. Patients meeting the following criteria were enrolled: (i) Histologically confirmed SCCHN, (ii) previous cisplatin-based chemotherapy either as definitive chemoradiotherapy or induction chemotherapy or post-operative chemoradiotherapy and (iii) documented R/M disease after platinum-based chemotherapy given as part of initial treatment. For this study, appropriate approvals were obtained from the institutional review committee.

These patients received oral MTX $10 \mathrm{mg}$ once a day, 4 days a week. To contain the possible side effects associated with MTX, folinic acid $15 \mathrm{mg}$ per oral every 6 hourly on day 5 was prescribed. MTX was continued till progression of disease. After progression, few (2\%) of the patients were irradiated. Pre-treatment evaluation included medical history, physical examination, laboratory tests, computed tomography (CT) and/or magnetic resonance imaging (MRI) system. Response assessment was done on two monthly basis. Response was evaluated by CT or MRI according to the Response Evaluation Criteria in Solid Tumours version 1.0 or clinically. Response was categorised as complete response (CR), partial response (PR), stable disease (SD) or progressive disease (PD) in this study. Adverse events were evaluated according to the National Cancer Institute Common Toxicity Criteria version 3.0.

Progression-free survival (PFS) was calculated from the date of the first administration of oral MTX to the earliest date when the treatment was discontinued for PD. If the treatment was discontinued due to reasons other than PD, PFS was calculated from the date of the first administration of oral MTX to the date when the disease worsened or when the patient died from any cause. OS time was calculated from the date of the first administration of oral MTX to the date of death from any cause or to the last date of confirmed survival. Survivals were analysed by the Kaplan-Meier method. 
Table 1: Patient characteristics

\begin{tabular}{|c|c|}
\hline Patient characteristics & $n(\%)$ \\
\hline Median age: Years (range) & $51(22-75)$ \\
\hline \multicolumn{2}{|l|}{ Gender } \\
\hline Male & $52(73)$ \\
\hline Female & $19(27)$ \\
\hline \multicolumn{2}{|l|}{ Site distribution } \\
\hline Oral cavity & $41(58)$ \\
\hline Nasopharynx & $18(25)$ \\
\hline Hypopharynx & $05(07)$ \\
\hline Paranasal sinuses & $04(06)$ \\
\hline Larynx & $02(03)$ \\
\hline Oropharynx & $01(01)$ \\
\hline \multicolumn{2}{|l|}{ Clinical T-stage } \\
\hline T1 & $01(01)$ \\
\hline $\mathrm{T} 2$ & $07(10)$ \\
\hline T3 & $22(31)$ \\
\hline $\mathrm{T} 4$ & $41(58)$ \\
\hline \multicolumn{2}{|l|}{ Clinical N stage } \\
\hline NO & $26(22)$ \\
\hline $\mathrm{N}+$ & $45(78)$ \\
\hline \multicolumn{2}{|l|}{ Grade } \\
\hline Well & $24(34)$ \\
\hline Moderate & $29(41)$ \\
\hline Poor & $16(23)$ \\
\hline Undifferentiated & $02(03)$ \\
\hline \multicolumn{2}{|l|}{ Initial treatment modality } \\
\hline $\mathrm{CRT}^{*}$ & $07(10)$ \\
\hline $\mathrm{C}+\mathrm{CRT}^{+}$ & $57(80)$ \\
\hline $\mathrm{C}+\mathrm{S}+\mathrm{RT}^{\ddagger}$ & $03(04)$ \\
\hline $\mathrm{C}+\mathrm{S}+\mathrm{CRT} \mathrm{T}^{\S}$ & $04(06)$ \\
\hline
\end{tabular}

${ }^{*} \mathrm{CRT}$ : Definitive chemoradiotherapy, ${ }^{+} \mathrm{C}+\mathrm{CRT}$ : Induction chemotherapy followed by chemoradiotherapy, ${ }^{\circ} \mathrm{C}+\mathrm{S}+\mathrm{RT}$ : Induction chemotherapy followed by surgery and radiotherapy, ${ }^{5} \mathrm{C}+\mathrm{S}+\mathrm{CRT}$ : Induction chemotherapy followed by surgery and chemoradiotherapy

\section{Results}

Between June 2008 and December 2012, a total of 71 patients with $\mathrm{R} / \mathrm{M}$ SCCHN were treated with oral MTX according to the above treatment schedule at Shaukat Khanum Memorial Cancer Hospital and Research Centre, Lahore, Pakistan. All the patients had very low socioeconomic status and ECOG PS 1-3. With a median age of 51 years (range 22-75), there were $73 \%$ of males and $27 \%$ of females. All the patients were previously given cisplatin-based chemotherapy either as definitive chemoradiotherapy or induction chemotherapy or post-operative chemoradiotherapy. Site distribution was as oral cavity $58 \%$, nasopharynx $25 \%$, hypopharynx $7 \%$, paranasal sinuses $6 \%$, larynx $3 \%$ and oropharynx $1 \%$. Patterns of recurrence were as follows: Local 32\%, regional 7\%, locoregional 10\%, distant 15\% and persistent disease in $36 \%$ of the patients. $89 \%$ of the patients had T3-T4 disease and 74\% of the patients had node-positive disease at the time of presentation. Table 1 summarises the patient characteristics. Initial treatment schedule was as follows: 7 (10\%) patients were treated with definitive chemoradiotherapy, $57(80 \%)$ with induction chemotherapy followed by chemoradiotherapy, 3 (4\%) with induction chemotherapy followed by surgery and radiotherapy and 4 patients $(6 \%)$ with induction chemotherapy followed by surgery and chemoradiotherapy.

The toxicity noted was minimal. None of the patient had Grade 4 mucositis or haematological toxicity. 64 patients (90\%) had Grade 0, 1 or 2 while $7(10 \%)$ patients had Grade 3 mucositis. In haematological toxicity, only one patient had Grade 3 neutropenia while 11\% of patients had Grades 1 or 2 neutropenia. It is very notable that most of the patients $(88 \%)$ had no haematological toxicity at all. The details of other toxicities are shown in Table 2.

The response rates at the end of 2 months are depicted in Table 3. The most common cause of discontinuation of chemotherapy was progression of disease (49 patients [69\%]) and intolerable side effects in $5(7 \%)$. Symptom control was seen $55(77 \%)$ patients. The median follow-up was 5 months. The crude median PFS was 5 months. The Kaplan-Meir graph for estimated PFS is depicted in Figure 1.

In this study, we used oral MTX (10 mg once a day, 4 days a week) and it appeared to have promising signs of efficacy and an acceptable safety profile for the treatment of platinum-refractory or relapsed SCCHN patients. Very few of the platinum-refractory or relapsed SCCHN patients are suitable for salvage surgery or reirradiation. Most of them qualify for palliative chemotherapy. In these patients, therapeutic options are modest, and the duration of response and survival are usually short. Attempts have made to improve survival of these patients with different chemotherapy agents such as taxanes (docetaxel and 
Table 2: Toxicity related to oral MTX

\begin{tabular}{l|c|c|c|c|c}
\hline Grade & $\mathbf{0}$ & $\mathbf{1}$ & $\mathbf{2}$ & $\mathbf{3}$ & $\mathbf{4}$ \\
\hline Mucositis & $55(76)$ & $7(9)$ & $2(3)$ & $7(10)$ & 0 \\
\hline Anaemia & $69(97)$ & $2(3)$ & - & - & - \\
\hline Neutropenia & $62(87)$ & $6(9)$ & $2(2)$ & $1(1)$ & - \\
\hline Thrombocytopenia & $68(96)$ & $3(4)$ & - & - & - \\
\hline Febrile neutropenia & - & - & - & - & - \\
\hline Vomiting & - & - & - & - & - \\
\hline
\end{tabular}

MTX: Methotrexate

Table 3: Response rate at the end of 2 months $n$ (\%)

\begin{tabular}{l|l}
\hline$C R$ & $02(03)$ \\
\hline$P R$ & $03(04)$ \\
\hline$S D$ & $08(11)$ \\
\hline$P D$ & $58(82)$ \\
\hline
\end{tabular}

CR: Complete response, PR: Partial response, SD: Stable disease, PD: Progressive disease

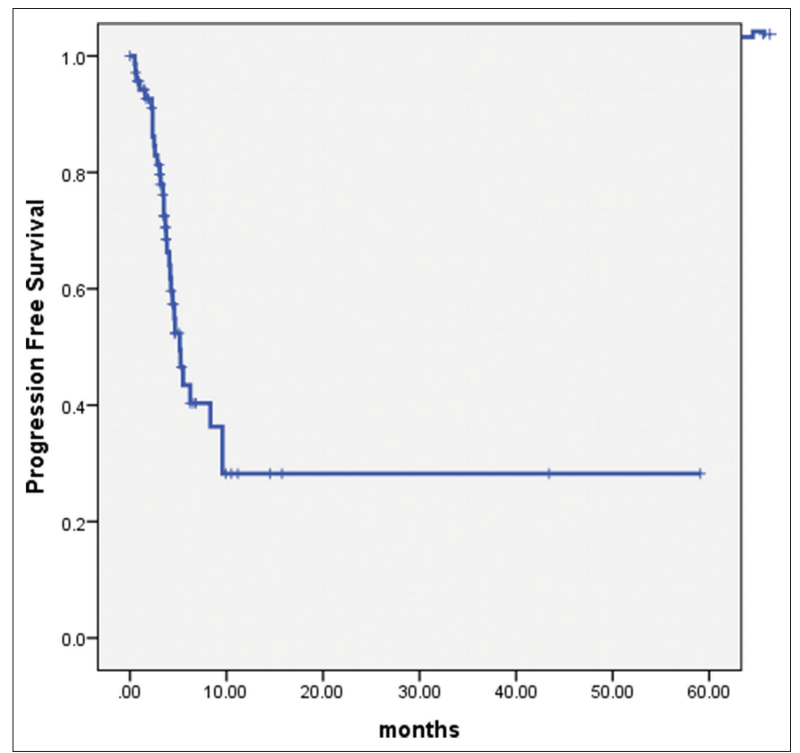

Figure 1: Progression-free survival

paclitaxel), MTX, bleomycin, capecitabine, ifosfamide, vinorelbine, irinotecan, S-1 and cetuximab. However, none of them showed survival advantage over others except cetuximab. ${ }^{[3,7,9,11,12,13]}$ The rate of response to the second-line chemotherapy was shown to be largely dependent on the therapy regimen given and on the treatment-free interval between initial and subsequent treatment. ${ }^{[16]}$ Herbst et al. ${ }^{[15]}$ reported the results of a cetuximab and cisplatin regimen and found that median OS was 4.3 months, and response rate was $13 \%$ in patients with documented PD or recurrence within 3 months of platinum-based therapy. Baselga et al.$^{[16]}$ showed a median OS of 5.0 months with a response rate of $11 \%$ in platinumrefractory patients treated with cetuximab followed by platinum chemotherapy. Yokota et al. ${ }^{[17]}$ reported the results of S1 monotherapy in SCCHN patients after the failure of platinum-based chemotherapy. In this study, the median PFS was 4.9 months and the median OS was 13.2 months. In our study, median PFS was 5 months.

The primary aim of treatment in palliative setting in platinum-refractory or relapsed SCCHN is to provide symptomatic relief and secondary aim is to improve OS. ${ }^{[18]}$ In LMICs like ours where the majority of the patients are from a lower socioeconomic background with poor nutrition, the use of therapies which are suitable to this population is warranted. Cetuximab is currently considered standard of care in platinum-refractory or relapsed patients with survival benefit and acceptable toxicity; however, its use is hampered in our country due to financial and logistical issues. Although taxanes have better response but at the cost of increased toxicity. ${ }^{[9]}$ MTX, most economical and least toxic, appears to be the best alternative in this situation. Moreover, patients who are not willing for intravenous MTX and who are not able to come to hospital from remote areas of the country on weekly basis for intravenous MTX due to poor execution status or financial considerations, oral MTX can be considered an equally effective agent in these patients.

\section{Conclusion}

Oral MTX is a simple, cost-effective and well-tolerated regimen to be used on outpatient basis for palliation 
in platinum-refractory or relapsed SCCHN in LMICs and debilitated patients. This treatment merits further evaluation in large-scale clinical trials.

\section{Conflict of Interest}

The authors declare that they have no conflict of interest.

\section{References}

1. Stewart BW, Kleihues PE. World Cancer Report. Lyon, France: International Agency for Research on Cancer Press; 2003.

2. Lag R, Melbert D, Krapcho M. SEER Cancer Statistics Review, 1975-2004. Bethesda, MD: National Cancer Institute; 2006.

3. Vermorken JB, Mesia R, Rivera F, et al. Platinum-based chemotherapy plus cetuximab in head and neck cancer. N Engl J Med 2008;359:1116-27.

4. Forastiere AA, Metch B, Schuller DE, et al. Randomized comparison of cisplatin plus fluorouracil and carboplatin plus fluorouracil versus methotrexate in advanced squamous-cell carcinoma of the head and neck: A southwest oncology group study. J Clin Oncol 1992;10:1245-51.

5. Cohen EE, Lingen MW, Vokes EE. The expanding role of systemic therapy in head and neck cancer. J Clin Oncol 2004;22:1743-52.

6. Jacobs C, Lyman G, Velez-García E, et al. A phase III randomized study comparing cisplatin and fluorouracil as single agents and in combination for advanced squamous cell carcinoma of the head and neck. J Clin Oncol 1992;10:257-63.

7. Burtness B, Goldwasser MA, Flood W, et al. Phase III randomized trial of cisplatin plus placebo compared with cisplatin plus cetuximab in metastatic/recurrent head and neck cancer: An eastern cooperative oncology group study. J Clin Oncol 2005;23:8646-54.

8. Colevas AD. Chemotherapy options for patients with metastatic or recurrent squamous cell carcinoma of the head and neck. J Clin Oncol 2006;24:2644-52.

9. Vermorken JB, Specenier P. Optimal treatment for recurrent/metastatic head and neck cancer. Ann Oncol 2010;21 Suppl 7:7252-61.

10. Price KA, Cohen EE. Current treatment options for metastatic head and neck cancer. Curr Treat Options Oncol 2012;13:35-46.

11. Vermorken J, Hitt R, Geoffrois L, et al. Cetuximab plus platinum-based therapy first-line in recurrent and/or metastatic $(\mathrm{R} / \mathrm{M})$ squamous cell carcinoma of the head and neck (SCCHN): Efficacy and safety results of a randomized phase IIItrial (EXTREME). Eur J Cancer 2007;5:324.

12. Tejani MA, Cohen RB, Mehra R. The contribution of cetuximab in the treatment of recurrent and/or metastatic head and neck cancer. Biologics 2010;4:173-85.

13. Rivera F, García-Castaño A, Vega N, et al. Cetuximab in metastatic or recurrent head and neck cancer: The EXTREME trial. Expert Rev Anticancer Ther 2009;9:1421-8.

14. Leon X, Hitt R, Constenla M, et al. A retrospective analysis of the outcome of patients (pts) with recurrent or metastatic squamous cell carcinoma of the head and neck ( $\mathrm{R}$ and M SCCHN) who are progressing while on a platinumbased palliative chemotherapy. Proc Am Soc Clin Oncol 2003;22:502.

15. Herbst RS, Arquette M, Shin DM, et al. Phase II multicenter study of the epidermal growth factor receptor antibody cetuximab and cisplatin for recurrent and refractory squamous cell carcinoma of the head and neck. J Clin Oncol 2005;23:5578-87.

16. Baselga J, Trigo JM, Bourhis J, et al. Phase II multicenter study of the antiepidermal growth factor receptor monoclonal antibody cetuximab in combination with platinum-based chemotherapy in patients with platinum-refractory metastatic and/or recurrent squamous cell carcinoma of the head and neck. J Clin Oncol 2005;23:5568-77.

17. Yokota T, Onozawa Y, Boku N, et al. S-1 monotherapy for recurrent or metastatic squamous cell carcinoma of the head and neck after progression on platinum-based chemotherapy. Jpn J Clin Oncol 2011;41:1351-7.

18. Murthy V, Gupta T, Agarwal JP, et al. Cautious optimism in advanced incurable head neck cancer. Radiother Oncol 2008;89:123-4. 